\title{
Metallorganische Verbindungen der Lanthanoide, 63 [1] \\ Pentalithium-okta-tert-butoxy-samarat(III): Synthese und Röntgenstrukturanalyse eines aus zwei flächenverknüpften Würfeln aufgebauten Lanthanoidclusters
}

\author{
Organometallic Compounds of the Lanthanoides, 63 [1] \\ Pentalithium Octa(tert-butoxy)samarat(III): Synthesis and X-Ray Structural Analysis \\ of a Lanthanoid Cluster Made by Two Face Linked Cubes \\ Herbert Schumann*, Gabriele Kociok-Köhn, Andreas Dietrich und Frank H. Görlitz \\ Institut für Anorganische und Analytische Chemie der Technischen Universität Berlin, \\ Straße des 17. Juni 135, W-1000 Berlin 12
}

Herrn Prof. Dr. E. Weiß zum 65. Geburtstag gewidmet

Z. Naturforsch. 46b, 896-900 (1991); eingegangen am 28. Dezember 1990

Pentalithium Octa(tert-butoxy)samarat(III), Synthesis, X-Ray

$[\mathrm{Li}(\mathrm{tmed})]_{3}\left[\mathrm{Sm}\left(\mathrm{CH}_{3}\right)_{6}\right]$ reacts with tert-butanol in diethyl ether to give $\left[\mathrm{Li}_{5} \mathrm{Sm}\left(\mathrm{O}-t-\mathrm{C}_{4} \mathrm{H}_{9}\right)_{8}\right]$ (1), the structure of which has been elucidated by X-ray structure analysis. 1 crystallizes tetragonally with $a=1703(4)$ and $c=3178(1) \mathrm{pm}$, space group $\mathrm{P} 4_{1} 2_{1} 2, Z=8$. The structure was refined to $R=0.042$ for 4082 observed reflections $\left(\mathrm{F}_{\mathrm{o}}>6 \sigma\left(\mathrm{F}_{\mathrm{o}}\right)\right)$.

\section{Einleitung}

Die Alkoxide der dreiwertigen Elemente der 3. Nebengruppe und der Lanthanoide werden gemeinhin mit der Formel $\mathrm{M}(\mathrm{OR})_{3}$ beschrieben [2] und auch so in zahlreichen Chemikalienkatalogen, beispielsweise als Ausgangsmaterialien für (über Sol-Gel-Prozesse zu gewinnende) Spezialkeramiken, feilgeboten. Daß dies nicht immer richtig ist, zeigte die Röntgenstrukturanalyse des vermeintlichen Yttrium-tri-iso-propylates, das als tetragonal-monopyramidaler Cluster mit interstitiellem Sauerstoff, $\left[\left(i-\mathrm{C}_{3} \mathrm{H}_{7} \mathrm{OY}\right)_{5}\left(\mu-\mathrm{O}-i-\mathrm{C}_{3} \mathrm{H}_{7}\right)_{8}\left(\mu^{5}-\mathrm{O}\right)\right]$ [3], kristallisiert, sowie die röntgenographische Charakterisierung der Lanthanoidalkoxide

$\left[\mathrm{Nd}_{6}\left(\mathrm{O}-i-\mathrm{C}_{3} \mathrm{H}_{7}\right)_{17}\left(\mu^{6}-\mathrm{Cl}\right)\right][4]$,

$\left[\left(\mathrm{C}_{5} \mathrm{H}_{5} \mathrm{Gd}\right)_{5}\left(\mu-\mathrm{OCH}_{3}\right)_{8}\left(\mu^{5}-\mathrm{O}\right)\right][5]$ und

$\left[\mathrm{Na}_{2}\left(t-\mathrm{C}_{4} \mathrm{H}_{9} \mathrm{OGd}\right)_{4}\left(\mu-\mathrm{O}-t-\mathrm{C}_{4} \mathrm{H}_{9}\right)_{8}\left(\mu^{6}-\mathrm{O}\right)\right]$ [5]. Monomere Alkoxide $\mathrm{Ln}(\mathrm{OR})_{3}$ werden offensichtlich nur bei Verwendung sterisch so anspruchsvoller Reste $\mathrm{R}$ wie 2,6-Di-tert-butylphenyl [6,7], 2,6-Ditert-butyl-4-methyl-phenyl [8] oder 2,6-Di(phenyl)phenyl [9] erhalten.

Um zu prüfen, ob diese Clusterbildung auf die bisher ausnahmslos als Ausgangsmaterial verwendeten Lanthanoidchloride zurückzuführen ist,

* Sonderdruckanforderungen an Prof. Dr. H. Schumann.

Verlag der Zeitschrift für Naturforschung, D-7400 Tübingen 0932-0776/91/0700-0896/\$01.00/0 ersetzten wir diese durch die sehr reaktiven homoleptischen Hexamethyllanthanoid-Komplexe $[\mathrm{Li}(\mathrm{tmed})]_{3}\left[\mathrm{Ln}\left(\mathrm{CH}_{3}\right)_{6}\right][10]$, von denen bekannt ist, daß sie mit Alkoholen Methan bilden [11].

\section{Ergebnisse und Diskussion}

Synthese und Eigenschaften

Tris[(tetramethylethylendiamin)lithium]hexamethylsamarat(III) reagiert in Diethylether bei $0{ }^{\circ} \mathrm{C}$ mit tert-Butanol augenblicklich unter Entwicklung von Methan. Beim Konzentrieren der auf $-78^{\circ} \mathrm{C}$ abgekühlten Lösung bilden sich farblose Kristalle von Pentalithium-okta-tert-butoxysamarat(III) (1):

$$
\begin{aligned}
& {[\mathrm{Li}(\mathrm{tmed})]_{3}\left[\mathrm{Sm}\left(\mathrm{CH}_{3}\right)_{6}\right] \stackrel{+t-\mathrm{C}_{4} \mathrm{H}_{9} \mathrm{OH}}{\underset{-\mathrm{CH}_{4},-\mathrm{tmed}}{\longrightarrow}}} \\
& \underset{\mathbf{1}}{\left[\mathrm{Li}_{5} \mathrm{Sm}\left(\mathrm{O}-t-\mathrm{C}_{4} \mathrm{H}_{9}\right)_{8}\right]}
\end{aligned}
$$

1 sublimiert bei $135^{\circ} \mathrm{C} / 0,5$ Torr. Luftzutritt über mehrere Stunden bewirkt keine sichtbare Veränderung der Verbindung.

\section{Röntgenstrukturanalyse}

Die Röntgenstrukturanalyse von 1 zeigt einen $\mathrm{SmLi}_{5} \mathrm{O}_{6}$-Cluster in der Form zweier flächenverbundener primitiver Würfel (Abb. 1), der durch die an den Sauerstoffatomen gebundenen tert-Butylgruppen weitgehend abgeschirmt ist. Durch die 


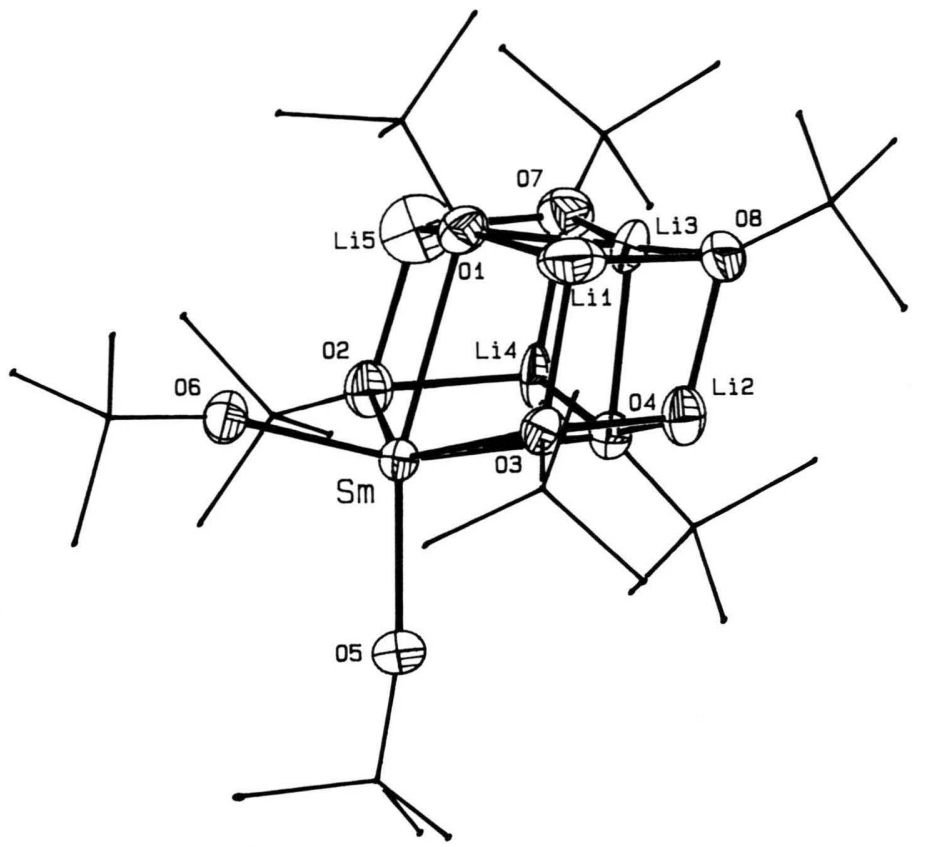

Abb. 1. Struktur von 1 im Kristall [19] (die tert-Butylgruppen sind aus Gründen der Übersicht durch Striche symbolisiert. Die C-Atome der tert-Butylgruppen sind entsprechend ihrer Stellung am O-Atom numeriert.) unterschiedliche Natur der Bindungspartner ist der Doppelwürfel - ein Strukturelement, das bisher noch nicht bei Lanthanoidalkoxiden gefunden wurde - stark verzerrt (Abb. 2). Die Winkel streuen daher stark um die idealen Winkel von $90^{\circ}$ bzw. $180^{\circ}$. Zwei an Samarium gebundene tert-Butylatgruppen sind nicht in den Cluster mit einbezogen und bewirken auf diese Weise den Erhalt der bereits in der Ausgangsverbindung vorliegenden oktaedrischen Koordination des Samariumatoms.
Die Sauerstoffatome der acht tert-Butylatgruppen zeigen unterschiedliche Bindungsarten: $O(5)$ und $\mathrm{O}(6)$ sind terminal an Samarium gebunden; $\mathrm{O}(7)$ und $\mathrm{O}(8)$ verbinden jeweils drei Lithiumatome des $\mathrm{Li}_{5}$-Gerüstes über $\mu^{3}$-Brücken $(\mathrm{O}(8)$ : $\mathrm{Li}(1), \mathrm{Li}(2)$, $\mathrm{Li}(3)$; $\mathrm{O}(7)$ : $\mathrm{Li}(3), \mathrm{Li}(4), \mathrm{Li}(5))$; $\mathrm{O}(2)$ und $\mathrm{O}(3)$ verbinden das Samarium und jeweils zwei Lithiumatome über $\mu^{3}$-Brücken $(\mathrm{O}(2)$ : $\mathrm{Sm}, \mathrm{Li}(4), \mathrm{Li}(5)$; $\mathrm{O}(3)$ : $\mathrm{Sm}, \mathrm{Li}(1), \mathrm{Li}(2)) ; \mathrm{O}(1)$ und $\mathrm{O}(4)$, diagonale Eckpunkte der gemeinsamen Fläche des Doppel-

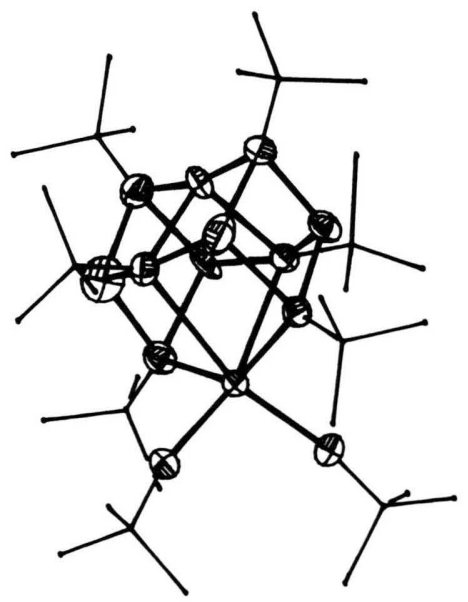

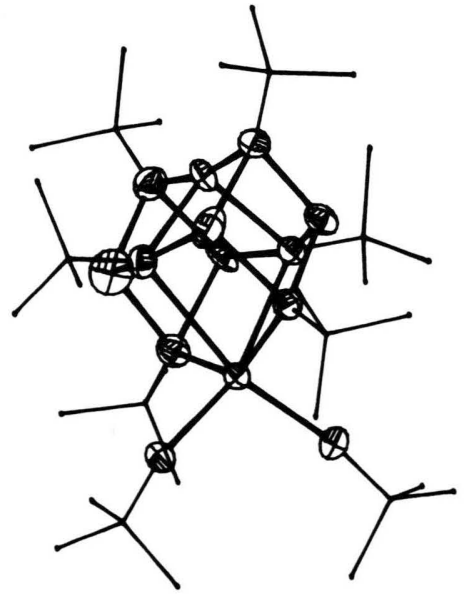

Abb. 2. Stereobild der Struktur von 1 (die H-Atome sind aus Gründen der Übersichtlichkeit weggelassen). 
würfels besetzend, zeigen eine seltene $\mu^{4}$-Bindung jeweils zu Samarium und drei der fünf Lithiumatome (O(1): $\mathrm{Sm}, \mathrm{Li}(1), \mathrm{Li}(3), \mathrm{Li}(5) ; \mathrm{O}(4)$ : $\mathrm{Sm}, \mathrm{Li}(2)$, $\mathrm{Li}(3), \operatorname{Li}(4))$. Dementsprechend sind auch die Sauerstoff-Samarium-Abstände unterschiedlich lang. Die kürzeste Bindung zeigen die endständig gebundenen tert-Butoxygruppen mit $c a .213 \mathrm{pm}$ $(\mathrm{Sm}-\mathrm{O}(5)=213,5(7) ; \mathrm{Sm}-\mathrm{O}(6)=213,7(7))$; mittlere Bindungslängen von $c a .238$ pm zeigen die $\mu^{3}$ verbrückenden Sauerstoffatome $(\mathrm{Sm}-\mathrm{O}(2)=$ 236,9(8); $\mathrm{Sm}-\mathrm{O}(3)=239,3(9))$, und erwartungsgemäß die größten Abstände zeigen die $\mu^{4}$-bindenden Atome $\mathrm{O}(1)$ und $\mathrm{O}(4)$ mit ca. $260 \mathrm{pm}(\mathrm{Sm}-\mathrm{O}(1)=$ 262,6(6) bzw. $\mathrm{Sm}-\mathrm{O}(4)=257,7(7))$.

Entsprechende Längenunterschiede zeigen auch die Li-O-Bindungen. Das $\mu^{4}$-bindende Li(3) zeigt im Mittel einen Abstand von 198 pm zu den Sauerstoffatomen $\mathrm{O}(1), \mathrm{O}(4), \mathrm{O}(7)$ und $\mathrm{O}(8)$, während die anderen, $\mu^{3}$-verbrückenden Lithiumatome ungefähr 188 pm von den an sie gebundenen Sauerstoffatomen entfernt sind. Es ist davon auszugehen, daß diese ungewöhnlichen Koordinationsverhältnisse nur durch die sperrigen tert-Butylgruppen ermöglicht werden, die die kleinen Lithiumatome vollständig abschirmen. Eine stereoskopische Darstellung der Elementarzelle (Abb. 3) läßt sowohl eine zweizählige als auch eine vierzählige Schraubenachse erkennen.

Eine ähnliche Variation in den Metall-Sauerstoff-Abständen in Abhängigkeit vom Verbrükkungsgrad der Sauerstoffatome findet man in Verbindungen anderer Lanthanoide. Samariumverbindungen, die für einen direkten Vergleich mit $\mathbf{1}$ geeignet wären, sind noch nicht bekannt. Man kann lediglich feststellen, daß der Abstand $\mathrm{Sm}-\mathrm{O}$ in
$\left(\mathrm{C}_{5} \mathrm{Me}_{5}\right)_{2} \mathrm{SmOC}_{6} \mathrm{HMe}_{4}[12] \mathrm{mit} 213 \mathrm{pm}$ den terminalen $\mathrm{Sm}-\mathrm{O}(5)$ - und $\mathrm{Sm}-\mathrm{O}(6)$-Abständen in $\mathbf{1}$ entspricht. Eine gleichlaufende Veränderung der Metall-Sauerstoff-Abstände wie in $\mathbf{1}$ findet man in den Verbindungen $\left[\mathrm{Nd}_{6}\left(\mathrm{O}-i-\mathrm{C}_{3} \mathrm{H}_{7}\right)_{17}\left(\mu^{6}-\mathrm{Cl}\right)\right]$ [4] mit $\mathrm{Nd}-\mathrm{O}($ terminal $)=205(2), \mathrm{Nd}-\mathrm{O}\left(\mu^{2}\right)=236(4)$ und $\mathrm{Nd}-\mathrm{O}\left(\mu^{3}\right)=245(5)$, in $\left[\mathrm{Y}_{4} \mathrm{Cl}_{2}\left(\mathrm{O}-t-\mathrm{C}_{4} \mathrm{H}_{9}\right)_{10} \mathrm{O}\right]$ [13] mit $\mathrm{Y}-\mathrm{O}$ (terminal) $=205, \mathrm{Y}-\mathrm{O}\left(\mu^{2}\right)=230$, $\mathrm{Y}-\mathrm{O}\left(\mu^{3}\right)=240$ und $\mathrm{Y}-\mathrm{O}\left(\mu^{4}\right)=246 \mathrm{pm}$, sowie in $\left[\left(i-\mathrm{C}_{3} \mathrm{H}_{7} \mathrm{OY}\right)_{5}\left(\mu-\mathrm{O}-i-\mathrm{C}_{3} \mathrm{H}_{7}\right)_{8}\left(\mu^{5}-\mathrm{O}\right)\right] \quad$ [3] $\mathrm{mit}$ $\mathrm{Y}-\mathrm{O}($ terminal $)=201, \mathrm{Y}-\mathrm{O}\left(\mu^{2}\right)=225, \mathrm{Y}-\mathrm{O}\left(\mu^{3}\right)$ $=227$ und $\mathrm{Y}-\mathrm{O}\left(\mu^{5}\right)=235$ pm, wobei für die beiden letzten Beispiele jeweils die gemittelten Abstände angegeben sind. Unter Berücksichtigung der Ionenradien in Abhängigkeit von der Koordinationszahl für $\mathrm{Sm}^{3+}(96 \mathrm{pm}), \mathrm{Nd}^{3+}(98 \mathrm{pm})$ und $\mathrm{Y}^{3+}(90 \mathrm{pm})$ [14] erhält man ein übereinstimmendes Bild bezüglich der Veränderungen der Lanthanoid-Sauerstoff-Bindungslängen.

\section{Experimentelles}

Alle Reaktionen wurden in einer Atmosphäre von trockenem, $\mathrm{O}_{2}$-freien Argon mit Hilfe der Schlenktechnik ausgeführt. Diethylether wurde über $\mathrm{Na}$ /Benzophenon getrocknet und unter Argon abdestilliert. [Li(tmed $]_{3}\left[\mathrm{Sm}\left(\mathrm{CH}_{3}\right)_{6}\right]$ wurde durch Umsetzung von $\mathrm{SmCl}_{3}$ (THF) $)_{2}$ mit $\mathrm{CH}_{3} \mathrm{Li}$ und Tetramethylethylendiamin (tmed) in Diethylether [10] dargestellt. tert-Butanol wurde durch Rückflußkochen über mit Iod aktivierten Magnesiumspänen absolutiert und anschließend abdestilliert.

In einem ausgeflammten und mit getrocknetem, Sauerstoff-freien Argon gefüllten Kolben werden $0,55 \mathrm{~g}(0,90 \mathrm{mmol})[\mathrm{Li}(\mathrm{tmed})]_{3}\left[\mathrm{Sm}\left(\mathrm{CH}_{3}\right)_{6}\right]$ in $50 \mathrm{ml}$ Ether suspendiert und auf $0{ }^{\circ} \mathrm{C}$ gekühlt. Innerhalb
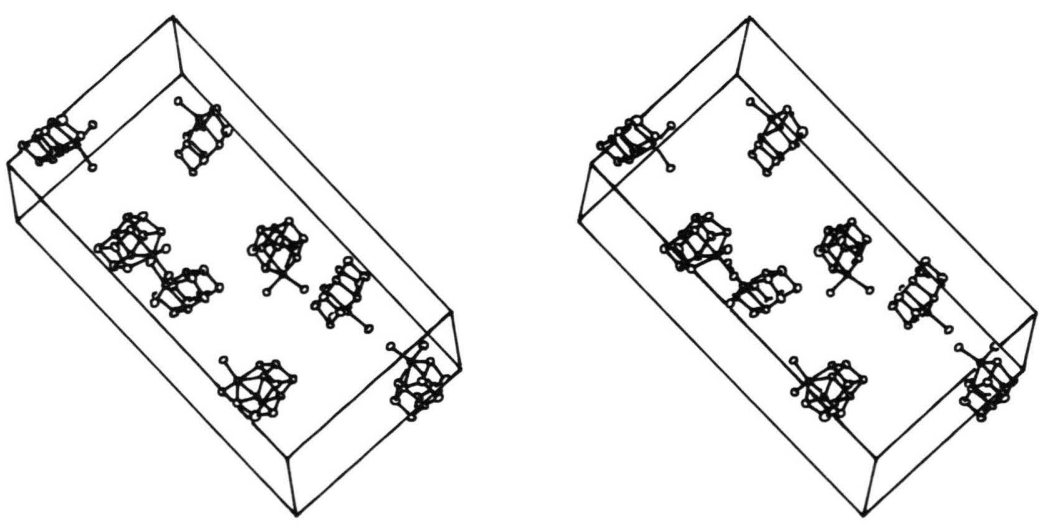

Abb. 3. Stereoskopische Darstellung der Elementarzelle von 1. Die asymmetrischen Einheiten zeigen der Übersichtlichkeit halber nur das $\mathrm{Li}_{5} \mathrm{SmO}_{8}$-Gerüst. 
von $30 \mathrm{~min}$ werden $0,51 \mathrm{ml}(5,4 \mathrm{mmol})$ tert-Butanol in $10 \mathrm{ml}$ Ether zugetropft. Anschließend beendet man die Kühlung und engt auf $10 \mathrm{ml}$ ein. Bei $-78{ }^{\circ} \mathrm{C}$ fallen im Verlauf von $12 \mathrm{~h}$ farblose Kristalle aus, die von der überstehenden Lösung durch Abdekantieren befreit werden. Ausbeute: $235 \mathrm{mg}(34 \%)$. IR-Spektrum ( $\left.\mathrm{KBr}, \mathrm{cm}^{-1}\right)$ : $450 \mathrm{st}$,

Tab. I. Atomkoordinaten und äquivalente isotrope Temperaturfaktoren $\left[\AA^{2}\right]$ in $\left[\mathrm{Li}_{5} \mathrm{Sm}\left(\mathrm{O}-t-\mathrm{C}_{4} \mathrm{H}_{9}\right)_{8}\right](\mathbf{1})$ mit Standardabweichungen in Klammern.

\begin{tabular}{|c|c|c|c|c|}
\hline Atom & $x$ & $y$ & $z$ & $\mathrm{~B}_{\mathrm{eq}}$ \\
\hline $\mathrm{Sm}$ & $0,6835(0)$ & $0,1868(0)$ & $0,4432(0)$ & 1,79 \\
\hline O 1 & $0,6976(4)$ & $0,1854(5)$ & $0,5255(2)$ & 2,46 \\
\hline $\mathrm{O} 2$ & $0,6278(4)$ & $0,3057(5)$ & $0,4679(3)$ & 2,71 \\
\hline $\mathrm{O} 3$ & $0,7929(4)$ & $0,1043(4)$ & $0,4599(2)$ & 2,14 \\
\hline O4 & $0,7966(4)$ & $0,2774(4)$ & $0,4661(2)$ & 2,04 \\
\hline O 5 & $0,7030(4)$ & $0,2043(5)$ & $0,3778(2)$ & 2,75 \\
\hline O6 & $0,5838(4)$ & $0,1107(5)$ & $0,4455(2)$ & 2,76 \\
\hline O7 & $0,7234(5)$ & $0,3523(5)$ & $0,5407(3)$ & 2,94 \\
\hline O 8 & $0,8699(4)$ & $0,1814(5)$ & $0,5318(3)$ & 2,80 \\
\hline Li 1 & $0,7880(15)$ & $0,1175(16)$ & $0,5199(8)$ & 3,98 \\
\hline $\mathrm{Li} 2$ & $0,8627(10)$ & $0,1860(13)$ & $0,4750(7)$ & 2,62 \\
\hline Li 3 & $0,7900(9)$ & $0,2620(10)$ & $0,5315(6)$ & 2,09 \\
\hline $\mathrm{Li} 4$ & $0,7223(11)$ & $0,3559(13)$ & $0,4836(7)$ & 2,57 \\
\hline Li 5 & $0,6448(16)$ & $0,2826(13)$ & $0,5250(9)$ & 4,02 \\
\hline C 11 & $0,6551(7)$ & $0,1415(7)$ & $0,5582(4)$ & 2,80 \\
\hline C 12 & $0,6641(9)$ & $0,0534(8)$ & $0,5506(4)$ & 4,53 \\
\hline C 13 & $0,6842(9)$ & $0,1622(9)$ & $0,5996(4)$ & 4,65 \\
\hline C 14 & $0,5688(8)$ & $0,1630(8)$ & $0,5539(4)$ & 4,04 \\
\hline C 21 & $0,5590(8)$ & $0,3482(7)$ & $0,4540(4)$ & 3,09 \\
\hline C 22 & $0,4914(9)$ & $0,3345(11)$ & $0,4823(5)$ & 5,55 \\
\hline C 23 & $0,5323(9)$ & $0,3150(11)$ & $0,4121(4)$ & 6,21 \\
\hline C 24 & $0,5778(10)$ & $0,4345(8)$ & $0,4527(5)$ & 5,76 \\
\hline C 31 & $0,8159(8)$ & $0,0341(7)$ & $0,4388(4)$ & 2,99 \\
\hline C 32 & $0,8392(12)$ & $-0,0228(10)$ & $0,4719(6)$ & 8,06 \\
\hline C 33 & $0,7543(9)$ & $0,0017(8)$ & $0,4119(4)$ & 4,25 \\
\hline C 34 & $0,8887(9)$ & $0,0514(10)$ & $0,4135(6)$ & 6,28 \\
\hline C 41 & 0,85 & $51(7)$ & $07(4)$ & 2,64 \\
\hline C 42 & $0,9118(8)$ & $0,3640(9)$ & $0,4715(5)$ & 5,03 \\
\hline C 43 & $0,8927(8)$ & $0,2750(8)$ & $0,4097(5)$ & 3,78 \\
\hline C 44 & $0,8036(9)$ & $0,3885(8)$ & $0,4164(4)$ & 4,34 \\
\hline C 51 & $0,7027(7)$ & $0,2101(7)$ & $0,3330(3)$ & 2,91 \\
\hline C 52 & $0,6213(10)$ & $0,1838(14)$ & $0,3184(5)$ & 9,51 \\
\hline C 53 & $0,7691(12)$ & $0,1546(12)$ & $0,3167(5)$ & 7,50 \\
\hline C 54 & $0,7173(14)$ & $0,2943(9)$ & $0,3194(5)$ & 7,89 \\
\hline C61 & $0,5199(8)$ & $0,0580(9)$ & $0,4373(4)$ & 4,12 \\
\hline C 62 & $0,5175(8)$ & $0,0372(9)$ & $0,3920(4)$ & 4,66 \\
\hline C 63 & $0,4420(7)$ & $0,0988(10)$ & $0,4478(5)$ & 5,14 \\
\hline C 64 & $0,5292(11)$ & $-0,0161(9)$ & $0,4639(6)$ & 7,06 \\
\hline C 71 & $0,7245(9)$ & $0,4126(8)$ & $0,5713(4)$ & 3,81 \\
\hline C 72 & $0,7443(11)$ & $0,4917(8)$ & $0,5514(6)$ & 6,96 \\
\hline C 73 & $0,7924(13)$ & $0,3966(12)$ & $0,6016(6)$ & 8,86 \\
\hline C 74 & $0,6479(12)$ & $0,4138(12)$ & $0,5948(6)$ & 9,21 \\
\hline C 81 & $0,9378(8)$ & $0,1644(8)$ & $0,5565(4)$ & 3,91 \\
\hline C 82 & $0,9540(10)$ & $0,2405(12)$ & $0,5829(6)$ & 7,62 \\
\hline C 83 & $0,9233(10)$ & $0,0912(10)$ & $0,5833(6)$ & 7,10 \\
\hline C 84 & $1,0021(9)$ & $0,1509(15)$ & $0,5244(6)$ & 9,76 \\
\hline
\end{tabular}

br, $500 \mathrm{st}, \mathrm{br}, 750 \mathrm{~s}, 860 \mathrm{~s}, 920 \mathrm{~s}, 1210 \mathrm{st}, 1240 \mathrm{~s}$, $1360 \mathrm{~s}, 1375 \mathrm{~s}, 1430 \mathrm{~m}$, br, $1470 \mathrm{~m}, 1570 \mathrm{~s}$, br, 1650 s, br, 2970 st, 3450 s, br; ${ }^{1} \mathrm{H}-\mathrm{NMR}(80 \mathrm{MHz}$, Bruker WP 80, $\mathrm{d}^{8}$-Toluol), $\delta$ [ppm] $=0,97,1,13$, $1,27,1,36,1,45 ;\left(\mathrm{CDCl}_{3}\right), \delta[\mathrm{ppm}]=0,95,1,17$ (breit), 1,28, 1,32, 1,38; ${ }^{13} \mathrm{C}-\mathrm{NMR}(20,15 \mathrm{MHz}$, Bruker WP 80, $\mathrm{d}^{8}$-Toluol), $\delta$ [ppm] $=35,28$ (breit), 35,48, 35,67, 36,13; MS (70 eV, $\left.135^{\circ} \mathrm{C}, \mathrm{M} / \mathrm{e}\right): 408$ $\left[\mathrm{Li}_{3} \mathrm{SmO}\left(\mathrm{OC}_{4} \mathrm{H}_{9}\right)_{3}\right]^{+}, 248\left[\mathrm{LiSmO}\left(\mathrm{OC}_{4} \mathrm{H}_{9}\right)\right]^{+}, 167$ $\left[\mathrm{Li}_{3}\left(\mathrm{OC}_{4} \mathrm{H}_{9}\right)_{2}\right]^{+}, 87\left[\mathrm{Li}_{2}\left(\mathrm{OC}_{4} \mathrm{H}_{9}\right)\right]^{+}$.

$$
\begin{aligned}
& \mathrm{C}_{32} \mathrm{H}_{72} \mathrm{Li}_{5} \mathrm{O}_{8} \mathrm{Sm}(770,06) \\
& \text { Ber. } \\
& \text { Gef. } 49,91 \text { H } 50,43, \\
& \text { Gef. } 5 \text { \% 9,33. }
\end{aligned}
$$

1 kristallisiert tetragonal mit den Gitterkonstanten $a=1703(4), c=3178(1)$ pm mit $Z=8$ Formeleinheiten in der Elementarzelle, Raumgruppe P $4{ }_{1} 2$ 2 (Nr. 92), V $=9211 \times 10^{-30} \mathrm{~m}^{3}, \varrho_{\text {ber. }}=1,11 \mathrm{~g} /$ $\mathrm{cm}^{3}$. Unter Verwendung eines automatischen Vierkreisdiffraktometers Enraf-Nonius CAD 4 wurden bei 230(5) $\mathrm{K}$ mit $\mathrm{Mo}-\mathrm{K}_{\alpha}$-Strahlung (Graphitmonochromator) nach der $\omega$-Scan-Methode die Intensitäten von 4570 unabhängigen Reflexen mit $2 \theta \leq 48^{\circ}$ gemessen. 4082 Reflexe mit Intensitäten $\mathrm{F}_{\mathrm{o}} \geq 6 \sigma\left(\mathrm{F}_{\mathrm{o}}\right)$ wurden für die Strukturbestimmung verwendet. Die Intensitäten wurden einer Lorentzund Polarisationskorrektur [15] sowie einer empirischen Absorptionskorrektur (DIFABS [16], min. 0,94, max. 1,06) unterzogen. Die Struktur wurde mit Patterson-Methoden [17] gelöst und mit Block-Matrix-Least-Squares [18] verfeinert $(R=$ $\left.0,042, R_{\mathrm{w}}=0,069\right)$. Die Nichtwasserstoffe wurden anisotrop, die Wasserstoffe isotrop verfeinert. Die Wasserstoffpositionen sind mit konstantem, isotropen Temperaturfaktor $\left(\mathrm{U}_{\text {iso }}=0,08 \AA^{2}\right)$ berechnet $\left(\mathrm{d}_{\mathrm{C}-\mathrm{H}}=95 \mathrm{pm}\right)$. In Tab. I sind die fraktionellen Atomkoordinaten angegeben, Tab. II zeigt ausgewählte Bindungsabstände und -winkel. Weitere

Tab. II. Ausgewählte Bindungsabstände (pm) und Win$\operatorname{kel}(\mathrm{Grad})$ in $\left[\mathrm{Li}_{5} \mathrm{Sm}\left(\mathrm{O}-t-\mathrm{C}_{4} \mathrm{H}_{9}\right)_{8}\right](\mathbf{1})$.

\begin{tabular}{lllr}
\hline Bindung & $\begin{array}{l}\text { Abstand } \\
(\text { pm) }\end{array}$ & Atome & $\begin{array}{r}\text { Winkel } \\
(\text { Grad })\end{array}$ \\
\hline $\mathrm{Sm}-\mathrm{O}(1)$ & $262,6(6)$ & $\mathrm{O}(4)-\mathrm{Sm}-\mathrm{O}(1)$ & $69,8(2)$ \\
$\mathrm{Sm}-\mathrm{O}(2)$ & $236,9(8)$ & $\mathrm{O}(5)-\mathrm{Sm}-\mathrm{O}(1)$ & $163,9(3)$ \\
$\mathrm{Sm}-\mathrm{O}(3)$ & $239,3(7)$ & $\mathrm{O}(4)-\mathrm{Li}(3)-\mathrm{O}(1)$ & $91,6(8)$ \\
$\mathrm{Sm}-\mathrm{O}(4)$ & $257,2(7)$ & $\mathrm{O}(3)-\mathrm{Li}(3)-\mathrm{O}(1)$ & $94,0(7)$ \\
$\mathrm{Sm}-\mathrm{O}(5)$ & $212,5(7)$ & $\mathrm{O}(7)-\mathrm{Li}(3)-\mathrm{O}(4)$ & $94,8(8)$ \\
$\mathrm{Sm}-\mathrm{O}(6)$ & $213,7(7)$ & $\mathrm{Li}(3)-\mathrm{O}(1)-\mathrm{Sm}$ & $99,0(6)$ \\
$\mathrm{Li}(3)-\mathrm{O}(1)$ & $205(2)$ & $\mathrm{Li}(3)-\mathrm{O}(4)-\mathrm{Sm}$ & $99,5(5)$ \\
$\mathrm{Li}(3)-\mathrm{O}(4)$ & $210(2)$ & $\mathrm{Li}(3)-\mathrm{O}(8)-\mathrm{Li}(1)$ & $83(1)$ \\
$\mathrm{Li}(3)-\mathrm{O}(7)$ & $193(2)$ & $\mathrm{Li}(3)-\mathrm{O}(8)-\mathrm{Li}(2)$ & $85,2(9)$ \\
$\mathrm{Li}(3)-\mathrm{O}(8)$ & $193(2)$ & $\mathrm{Li}(3)-\mathrm{O}(4)-\mathrm{Li}(2)$ & $77,7(8)$ \\
\hline
\end{tabular}


Einzelheiten zur Kristallstrukturbestimmung können beim Fachinformationszentrum Karlsruhe, Gesellschaft für wissenschaftlich-technische Information, W-7514 Eggenstein-Leopoldshafen 2, unter Angabe der Hinterlegungsnummer CSD 55414, der Autoren und des Zeitschriftenzitats angefordert werden.
Der Deutschen Forschungsgemeinschaft und dem Fonds der Chemischen Industrie sowie dem Bundesminister für Bildung und Wissenschaft Graduiertenkolleg ,Synthese und Strukturaufklärung niedermolekularer Verbindungen" - danken wir für finanzielle Unterstützung dieser Arbeit und für ein Doktoranden-Stipendium (G.K.K.).
[1] H. Schumann und A. Dietrich, J. Organomet. Chem. 401, C 33 (1991).

[2] R. C. Mehrotra und J. M. Batwara, Inorg. Chem. 9, 2505 (1970); D. C. Bradley und D. P. Gaur, Metal Alkoxides; Academic Press, New York (1987); K. G. Caulton und L. G. Hubert-Pfalzgraf, Chem. Rev. 90, 969 (1990) und in diesen Arbeiten zitierte Literatur.

[3] O.Poncelet, W. J. Sartain, L. G. Hubert-Pfalzgraf, K. Folting und K. G. Caulton, Inorg. Chem. 28, 263 (1989).

[4] R. A. Andersen, D. H. Templeton und A. Zalkin, Inorg. Chem. 17, 1962 (1978).

[5] H. Schumann, G. Kociok-Köhn und J. Loebel, Z. Anorg. Allg. Chem. 581, 69 (1990).

[6] H. A. Stecher, A. Sen und A. L. Rheingold, Inorg. Chem. 27, 1130 (1988).

[7] P. B. Hitchcock, M. F. Lappert und R. G. Smith, Inorg. Chim. Acta 139, 183 (1987).

[8] P. B. Hitchcock, M. F. Lappert und A. Singh, J. Chem. Soc. Chem. Commun. 1983, 1499.

[9] G. B. Deacon, S. Nickel, P. MacKinnon und E. R. T. Tiekink, Aust. J. Chem. 43, 1245 (1990).
[10] H. Schumann und J. Müller, Angew. Chem. 90, 307 (1978); Angew. Chem., Int. Ed. Engl. 17, 276 (1978).

[11] H. Schumann, J. Müller, N. Bruncks, H. Lauke, J. Pickardt, H. Schwarz und K. Eckart, Organometallics 3, 69 (1984).

[12] W. J. Evans, T. P. Hanusa und K. R. Levan, Inorg. Chim. Acta 110, 191 (1985).

[13] W. J. Evans und M. S. Sollberger, Inorg. Chem. 27, 4417 (1988).

[14] R. D. Shannon, Acta Crystallogr. A 32, 751 (1976).

[15] B. A. Frenz, Enraf-Nonius, SDP-Structure Determination Package, Version 3.0, Enraf-Nonius, Delft (1985).

[16] N. Walker und D. Stuart, Acta Crystallogr. A39, 158 (1983).

[17] G. M. Sheldrick, SHELXS-86, Program for Crystal Stucture Determination, Universität Göttingen (1986).

[18] G. M. Sheldrick, SHELX-76, Program for Crystal Structure Determination, University of Cambridge, England (1976).

[19] C. K. Johnson, ORTEP, Report ORNL-5138, Oak Ridge National Laboratory Tennessee, U.S.A. (1976). 